\title{
The easy-to-hard effect: Transfer along the dimension of orientation in the rat
}

\author{
THOMAS H. TURNEY \\ University of Nevada, Reno, Nevada 89507
}

\begin{abstract}
Long-Evans rats were trained on an easy simultaneous discrimination problem and then transferred to a hard discrimination problem. The discriminanda were different orientations of stripes. In Experiment I, transfer was intradimensional with stimulus generalization controlled. The easy-tohard effect was not found. In Experiment II transfer was intradimensional and either compatible (nonreversal) or incompatible (reversal). With compatible transfer, rats trained on a prior easy problem learned the hard problem faster than rats trained only on the hard problem. Persistent negative transfer was found with incompatible transfer. Attention was not supported as underlying the easy-to-hard effect. Explanations based on specific sources of intradimensional transfer, such as stimulus generalization or adaptation level, are suggested.
\end{abstract}

Studies of transfer effects in animals (Lawrence, 1952; Logan, 1966; Marsh, 1969; Singer, Zentall, \& Riley, 1969; Mackintosh and Little, 1970) and men (May \& MacPherson, 1971; Trabasso, 1963) have shown that prior training on an easy discrimination problem improves performance on a difficult problem. This has been called the easy-to-hard effect. The control group is trained on the hard problem only. The easy-to-hard group receives an equal amount of training; however, training is divided between the easy and hard problems. The easy-tohard group learns the hard discrimination faster than the control group. The conventional paradigm involves simultaneous discrimination problems with transfer along a single dimension.

Explanations of the easy-to-hard effect have relied primarily upon: (a) stimulus generalization (Logan, 1966) or (b) attentional processes (Lawrence, 1952). Various experimenters have emphasized one of these while relegating some effect to the other. The present study tested the two explanations by controlling stimulus generalization as a source of intradimensional transfer while allowing attentional processes to function.

The dimension used in the present study was the orientation of stripes. Points on the continuum of $180 \mathrm{deg}$ were selected as discriminanda. Physical properties of the discriminanda other than orientation were not varied. Data from a psychophysical study by Ely (1969) permitted construction of an ordinal scale of difficulty. On the basis of Ely's study, the discrimination problem of 0 vs. $90 \mathrm{deg}$ was the least difficult of any studied. Discriminations between 30 vs. $60 \mathrm{deg}$ and 60 vs. $120 \mathrm{deg}$ were of much greater difficulty. Discriminanda of 60 and

The author is indebted to R. Allen Gardner, Michael R. Pullen, and Susan A. Shodahl for their assistance. The author's present address: Department of Psychology, New Mexico Tech, Socorro, New Mexico 87801.
$120 \mathrm{deg}$ may also be viewed as 60-deg angles of opposite slope ( +60 and $-60 \mathrm{deg})$.

The use of an orientation dimension made possible the control of excitatory (E) and inhibitory (I) habit strength in a manner differing from that used by Singer et al. (1969), who attempted to neutralize the effects of $E$ and $I$ by training on a three-discriminanda easy problem prior to transfer to the two-discriminanda hard problem. The present study avoided the difficulties encountered in their procedure by training on a two-discriminanda easy problem. Training on 0 vs. $90 \mathrm{deg}$, the discriminanda of the easy problem, would presumably generate gradients about these values. The discriminanda of the hard problem in Experiment I, +60 vs. $-60 \mathrm{deg}$, lie on the orientation dimension in a manner which made the net $\mathrm{E}$ and I from the easy problem equally strong at these points. Should 0 deg be the $\mathrm{S}+$, then $\mathrm{E}$ would be the same for both +60 - and -60 -deg angles, because both are equidistant from 0 deg. Likewise, if a 90-deg angle was $S-$, then I was equal at +60 - and -60 -deg angles. Generalization gradients as postulated by Logan (1966) are effectually neutralized. The subject should have no way of choosing $\mathrm{S}+$ from $\mathrm{S}-$ on the basis of differential response strength.

\section{EXPERIMENT I}

Experiment I proposed to assess the contributions of stimulus generalization and attention to the easy-to-hard effect. A simultaneous discrimination procedure was used with rats. The easy discrimination problem was defined as 0 vs. $90 \mathrm{deg}$, the hard discrimination problem as +60 vs. $-60 \mathrm{deg}$. Since transfer was intradimensional, an easy-to-hard effect would be predicted by attention theory. Stimulus generalization was controlled by the method discussed above. 
Counterbalancing for Experiment I required six conditions. Each discriminanda of the easy and hard problems served as $\mathrm{S}+$ for one half of the subjects being trained on that problem. The Easy-to-Hard group was also counterbalanced between problems. When Easy-to-Hard group subjects trained on the easy problem with $0 \mathrm{deg}$ as $\mathrm{S}+$ were transferred to the hard problem, one half received $+60 \mathrm{deg}$ as $\mathrm{S}+$ and the other half received $-60 \mathrm{deg}$ as $\mathrm{S}+$. Likewise, subjects trained on the easy problem with $90 \mathrm{deg}$ as $\mathrm{S}-$ received either +60 or $-60 \mathrm{deg}$ as $\mathrm{S}+$ on the hard problem. Eight subjects were randomly assigned to each condition.

The control group received 16 days of training on the hard problem alone. The Easy-to-Hard group received 6 consecutive days of training on the easy problem followed by 10 days of training on the hard problem. The transfer was made on the 7 th day because previous experimentation (Ely, 1969) had shown that at this time subjects on the easy problem responded at approximately $76 \%$ correct while subjects on the hard problem responded at approximately $50 \%$ correct. Thus, the former group appeared to have acquired a correct response while the latter was not responding above chance.

\section{Method}

Subjects. Sixty-one male, pigmented, Long-Evans rats served as subjects. When purchased from Simonson Laboratories, Gilroy, California, the subjects were approximately 51 days old and $220 \mathrm{~g}$ in weight. Of these, six were discarded for failure to meet pretraining criteria. Seven subjects were randomly discarded in order to equalize groups. The extra subjects were trained in order to offset any possible losses of subjects. Two subjects were discarded due to experimenter error.

Apparatus. The discrimination enclosure, measuring $60.9 \mathrm{~cm}$ long $\times 22.9 \mathrm{~cm}$ wide $\times 30.5 \mathrm{~cm}$ high, consisted of two choice compartments connected by a narrow passageway. Two transparent plastic panels were located in each compartment at opposite ends of the enclosure. Four In-Line Read-Out projectors mounted outside the enclosure displayed the discriminanda so that each was visible through the transparent panels. Each panel was hinged at the top so that when the subject exerted slight pressure outward from the compartment an independent microswitch was closed. A panel press on one of the two illuminated panels turned off the corresponding projectors and simultaneously turned on the projectors in the opposite compartment. A bead of water delivered beneath the panels automatically rewarded a correct choice.

A second enclosure used for preliminary training had the same basic floor plan. However, a single translucent panel illuminated by a miniature lamp was located in each compartment. $A$ bead of water was delivered beneath each panel whenever a lighted panel was pressed. Pressing a lighted panel simultaneously deactivated that panel and activated the panel in the opposite compartment.

The discrimination and preliminary training enclosures were housed separately in darkened rooms. A sound screen of white noise was provided during all phases of training.

Procedure. Throughout the experiment, subjects were housed individually. The subjects had access to dry Lab Chow and water for $24 \mathrm{~h}$ and $1 / 2 \mathrm{~h}$ per day, respectively.

Handling began on the first day following arrival of the subjects and consisted of a 3-min handling period per day devoted to each subject. Water deprivation also began at this time. Preliminary training began on the 11 th day following the 10 days of handling. On the first day of preliminary training the subjects were briefly shaped to press a continuously illuminated panel while restricted to one compartment of the preliminary training enclosure. Following shaping, 4 days were devoted to training the subjects to alternate between panels in opposite compartments (shuttle training). Pressing the panel turned off that panel and delivered a reward. Simultaneously, the panel in the opposite compartment was activated.

On the day following the last day of preliminary training, the subjects were switched to the discrimination enclosure and 16 days of discrimination training began. Each subject received a block of 24 trials on Day 1 of discrimination training and 72 trials on each remaining day. On each trial, the subject was presented two discriminanda (one on each panel) in one choice compartment on the discrimination enclosure. The subjects were consistently rewarded for selecting one of the discriminanda $(\mathrm{S}+)$ and not rewarded for selecting the other $(\mathrm{S}-)$ regardless of whether it appeared on the right or left panel. The $\mathbf{S}+$ and $S-$ occurred equally often on both the right and left panels.

The subjects in any problem were divided so that an angle serving as $S+$ for one half of the subjects served as $S$ - for the other half. At the end of each training session, the subject received immediate access to water in the home cage for $20 \mathrm{~min}$.

Discriminanda. Each projector displayed a circular field of three black and two white stripes measuring $1.27 \mathrm{~cm}$ in width. The field measured $6.35 \mathrm{~cm}$ in diameter. Different orientations of the field provide four discriminanda of 0 (horizontal), 60,90, and $120 \mathrm{deg}$. Angles of 60 and $120 \mathrm{deg}$ were also viewed as 60 -deg angles of opposite slope. The former was designated as positive (or right) and the latter as negative (or left).

\section{Results and Discussion}

The easy-to-hard effect was not demonstrated. Figure 1 displays the mean percent correct over all days in Experiment I. Statistical analysis was based on each subject's mean correct responses over the 10 posttransfer days. A fixed-effects 2 by 2 ANOVA showed no significant main effects or Easy Problem $\times$ Hard Problem interaction within counterbalanced experimental groups. These counterbalanced experimental groups were combined to form the

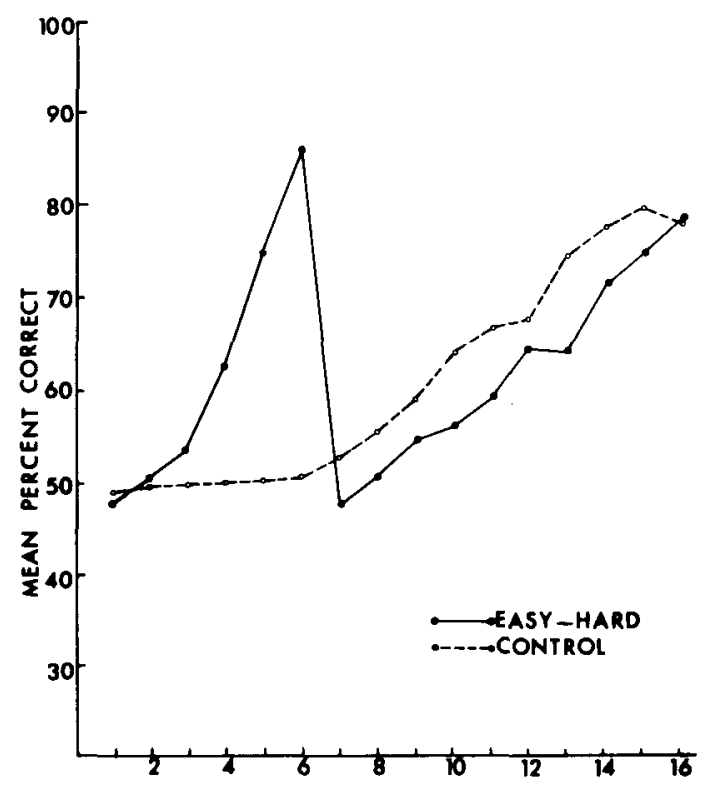

Figure 1. Mean percent correct responses during discrimination training in Experiment I. 
Easy-to-Hard group for the main comparison with the combined control group. The Easy-to-Hard group did not differ significantly from the control group $(\mathrm{t}=-1.876, \mathrm{df}=46, \mathrm{p}<0.1)$. The direction of the observed effect was the opposite of that expected from the easy-to-hard effect. A comparison between the two groups at the same point in training on the hard problem (i.e., Days 1 through 10 of the control group with Days 7 through 16 of the Easyto Hard group) shows some positive transfer, but the easy-to-hard effect requires that positive transfer be shown over the final 10 days of discrimination training for both groups. The solution of an easy problem was not a source of facilitation for a hard problem when stimulus generalization was controlled. This result clearly fails to support an attentional interpretation of the easy-to-hard effect.

\section{EXPERIMENT II}

Before we could conclude that the control of stimulus generalization eliminated the easy-to-hard effect, we had to demonstrate the easy-to-hard effect in rats with this procedure and apparatus. The second experiment permitted an easy-to-hard effect based on stimulus generalization.

Experiment II was identical to Experiment I, with a single exception: the discriminanda of the hard discrimination problem were +30 vs. $+60 \mathrm{deg}$. These discriminanda were employed for two reasons: (1) the angles lie between the discriminanda of the easy problem, 0 vs. $90 \mathrm{deg}$, and thus represent a conventional easy-to-hard paradigm on an orientation dimension; and (2) the difficulty of the problem was nearly equal to that of the hard discrimination problem $(+60,-60)$ in the first experiment (Ely, 1969).

Following Singer et al. (1969) the Easy-to-Hard group was partitioned into Compatible and Incompatible groups. The Compatible group received an $\mathrm{S}+$ on the hard problem that was nearer in steepness to the $\mathrm{S}+$ of the easy problem than to the $\mathrm{S}-$. The Incompatible group received an $\mathrm{S}+$ on the hard problem that was nearer to the $\mathrm{S}-$ of the easy problem. Thus, if the easy problem consisted of $0 \mathrm{deg}$ as $\mathrm{S}+$ and $90 \mathrm{deg}$ as $\mathrm{S}-$, transfer to the hard problem $(+30,+60)$ with $+30 \mathrm{deg}$ as $\mathrm{S}+$ would be compatible, while transfer with +60 as $S+$ would be incompatible.

For the Incompatible group, the steepness of $\mathrm{S}+$ and $\mathrm{S}-$ were reversed when they were transferred from the easy to the hard problem. For their Incompatible group, Singer et al. (1969) found immediate negative transfer, but this rapidly disappeared. Mackintosh and Little (1970), in replicating this finding, used it as a basis of questioning the role of stimulus generalization in the easy-to-hard effect. Mackintosh and Little reasoned that if stimulus generalization was functioning, then a stronger reversal effect should occur.

\section{Method}

Thirty-four male, pigmented, Long-Evans rats of the same age and weight as in Experiment I served as subjects. Of these, four rats were discarded for failure to meet pretraining criteria. The discriminanda were the same except that the hard discrimination problem was $+30 \mathrm{vs},+60 \mathrm{deg}$. The easy problem remained 0 vs. $90 \mathrm{deg}$. The apparatus and procedures were identical to those in Experiment I.

The control group received 16 days of training on the hard problem alone. The Easy-to-Hard groups received 6 consecutive days of training on the easy problem followed by 10 days of training on the hard problem. The Easy-to-Hard and control groups were counterbalanced within and between problems, as described in Experiment I. Counterbalancing inherently produced Compatible and Incompatible Easy-to-Hard groups as discussed above. Counterbalancing in Experiment II required six conditions. Five subjects were randomly assigned to each condition.

\section{Results and Discussion}

The Easy-to-Hard Compatible group demonstrated a powerful easy-to-hard effect. The Easy-to-Hard Incompatible group performed as if they had been reversed. Figure 2 displays the mean percent correct over all days in Experiment II. Statistical analysis was based on each subject's mean correct responses over the 10 posttransfer days. Dunn's multiple comparison procedure for planned nonorthogonal comparisons was employed for tests of significance (Kirk, 1968).

The Easy-to-Hard Compatible group performed significantly better than the control group $(p<.01)$; thus demonstrating the easy-to-hard effect with rats on an orientation dimension. The mean number of correct responses on the day of transfer to the hard problem (Day 7) does not differ significantly $(t=$

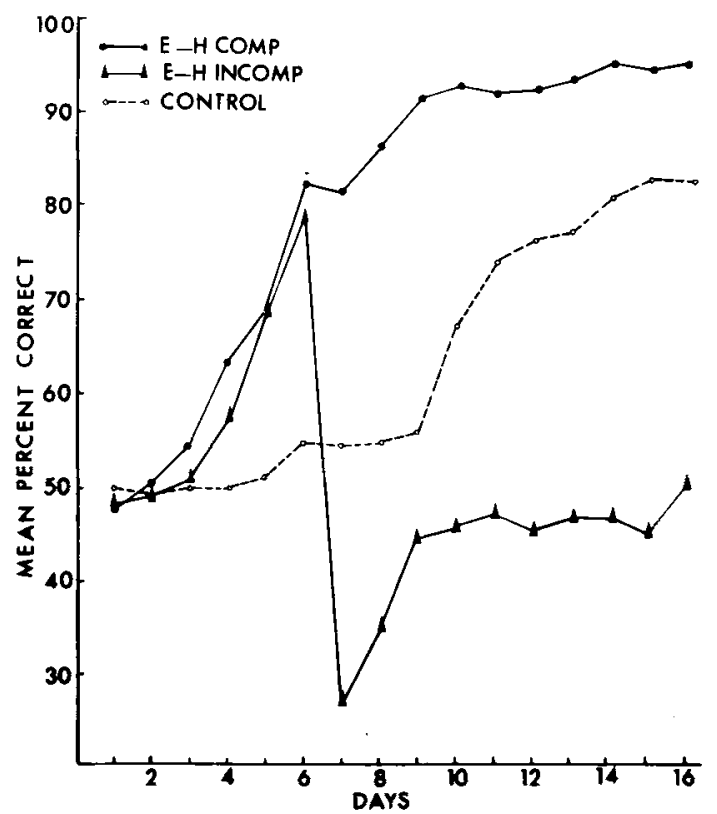

Figure 2. Mean percent correct responses during discrimination training in Experiment II. 
$.138, \mathrm{df}=18, \mathrm{p}>0.6)$ from the last day of training on the easy problem (Day 6).

Previous experimenters (Singer et al., 1969; Mackintosh \& Little, 1970) have found that immediate negative transfer in easy-to-hard incompatible groups rapidly disappeared. In the present experiment, the Easy-to-Hard Incompatible group initially performed as if reversed. Performance continued to be consistently poorer than that of the control group $(p<.01)$. This finding would be expected from a stimulus generalization position (Mackintosh \& Little, 1970).

\section{GENERAL DISCUSSION}

The present study extended the generality of the easy-to-hard effect to include transfer along an orientation dimension. The easy-to-hard effect was found with intradimensional transfer, but only when stimulus generalization was allowed to function. This result suggests that attentional processes did not contribute significantly to the easy-to-hard effect.

In Experiment II, powerful easy-to-hard and reversal effects were demonstrated with a conventional intradimensional transfer paradigm. The easy-to-hard effect in the Compatible group was demonstrated as a sustained high level of correct responding during transfer. From Logan's (1966) analysis based on generalized $E$ and $I$, it is unclear whether a sustained level or a noticeable decrement in correct responding should occur. The net difference in generalized response strength between the stimuli of the easy problem should be greater than between the stimuli of the hard problem. Transfer from stimuli of greater net difference between $E$ and I to stimuli of lesser difference would seem to produce a decrement in correct responding. The Compatible group of this experiment showed no significant decrement in mean number of correct responses on the day of transfer.

When transferred, the Incompatible group showed a strong preference for the discriminanda closest to the former $\mathbf{S}+$. The Incompatible group does not replicate the rapid recovery found by previous investigators (Singer et al., 1969; Mackintosh \& Little, 1970). Instead, persistent negative transfer was found for the duration of training on the hard problem. The persistence of negative transfer clearly fails to support the attentional interpretation of the easy-to-hard effect.

Explanations of the easy-to-hard effect other than stimulus generalization should not be overlooked. For example, an adaptation level approach (Capehart, Tempone, \& Hebert, 1969; James, 1953) might account for the present easy-to-hard effect in the following manner. Training to discriminate between stimuli on a dimension establishes an adaptation level near the psychophysical midpoint between the two stimuli. Training on a discrimination problem of 0 vs. 90 deg may establish an adaptation level near 45 deg. Furthermore, stimuli above adaptation level and below adaptation level are formed into distinct classes. Thus, the range of angles between 0 and 45 deg would form a class "less-steep." Angles between 45 and 90 deg would form a class "steeper." If subjects learned to respond to the stimulus class, i.e., to respond to the "steeper" (or "less-steep") member of a pair of stimuli, then the easy and hard problems would be the same problem. Perfect transfer with allowances for absolute discriminability should be found. In fact, nearly perfect transfer was found in the Easy-to-Hard Compatible group. Stimulus generalization does not clearly predict such a high level of facilitation.

An adaptation level approach could also explain the failure to find the easy-to-hard effect in Experiment I. After training on the easy problem, 0 vs. $90 \mathrm{deg}$, the discriminanda of the hard problem, +60 vs. $-60 \mathrm{deg}$, would both lie within the same stimulus class "steeper." No easy-to-hard facilitation would be predicted, and none was found. The adaptation level approach is only roughly formulated (see Riley, 1968), but such an approach deserves consideration along with stimulus generalization as a process involved in the easy-to-hard effect.

\section{REFERENCES}

Capehart, J., Tempone, V. J., \& Hebert, J. A theory of stimulus equivalence. Psychological Review, 1969, 76, 405-418.

ELY, D. Discrimination of stripes at different angles by the rat. Unpublished doctoral dissertation. University of Nevada, Reno, 1969.

JAMES, H. An application of Helson's theory of adaptation level to the problem of transposition. Psychological Review, $1953,60,345-352$.

KIRK, R. E. Experimental design: Procedures for the behavioral sciences. Belmont, CA: Brooks/Cole, 1968.

LAwRENCE, D. H. The transfer of a discrimination along a continuum. Journal of Comparative and Physiological Psychology, 1952, 45, 511-516.

Logan, F. A. Transfet of discrimination. Journal of Experimental Psychology, 1966, 71, 616-618.

Mackintosh, N. J., \& LiTtLE, L. An analys is of transfer along a continuum. Canadian Journal of Psychology, 1970, 24, 362-369.

Marsh, G. An evaluation of three explanations for the transfer of discrimination effect. Journal of Comparative and Physiological Psychology, 1969, 68, 268-275.

May, R. B., \& MacPherson, D. F. Size discrimination in children facilitated by changes in task difficulty. Journal of Comparative and Physiological Psychology, 1971, 75, 453-458.

RuLEY, D. A. Discrimination leaming. Boston: Allyn \& Bacon, 1968.

Singer, B., Zentall, T., \& Riley, D. A. Stimulus generalization and the easy-to-hard effect. Journal of Comparative and Physiological Psychology, 1969, 69, 528-535.

Trabasso, T. R. Stimulus emphasis and all-or-none learning in concept identification. Joumal of Experimental Psychology, $1963,65,398-406$.

(Received for publication April 29, 1975; revision accepted February 26, 1976.) 